\title{
Commission 15. (ÉTUDE PHYSIDUE DES COMÈTES)
}

I. La Commission recommande de publier une liste des photographies des comètes et de leurs spectres classées par ordre chronologique. La publication de cette liste sera faite trimestriellement, et a titre d'essai, aux annales de l'Observatoire de Meudon par les soins du Président.

Un appel sera adressé aux observatoires convenablement situés en longitude pour aider à combler les lacunes importantes que présentent actuellement les séries d'observation.

2. La Commission attire l'attention des observateurs sur la nécessité d'unifier les déterminations des magnitudes photographiques et visuelles des comètes afin de les rendre comparables entre elles. Elle les engage à approfondir la question de manière à pouvoir confronter les méthodes et choisir la meilleure, dans chaque cas, au prochain Congrès.

La même recommandation est faite pour la spectrophotométrie des comètes.

3. La Commission n'a pas la possibilité matérielle d'entreprendre elle-même la création d'un "Bureau des Comètes" et d'en assurer le fonctionnement. Mais elle encouragera tout observatoire ou astronome qui se chargerait de la discussion des observations obtenues sur une ou plusieurs comètes en lui facilitant l'envoi des documents photographiques dont il aura trouvé la liste dans la publication prévue au paragraphe $I$.

4. L'emploi de systèmes optiques à grand rapport d'ouverture ( $F / I$ par ex.) tels que la chambre de Schmidt est particulièrement recommandé pour l'étude photographique des comètes.

\section{Commission I6. (OBSERVATIONS PHYSIQUES DES PLANÈTES)}

I. La Commission recommande aux observateurs de Jupiter et de Saturne au télescope de s'attacher d'une manière plus systématique à l'étude des couleurs, des niveaux et des mouvements des taches de ces planetes.

2. La Commission exprime le vœu que les physiciens possédant l'équipement expérimental nécessaire entreprennent l'étude des équations d'état de gaz simples, en particulier l'hydrogène, l'hélium, le méthane et l'ammoniac jusqu'aux pressions les plus fortes que l'on puisse atteindre.

\section{Commission I8. (LoNGITUDES)}

La Commission mixte des longitudes a examiné les deux premiers fascicules de l'exposé d'ensemble de l'Opération Internationale des longitudes de r933. Elle est reconnaissante au Gouvernement Français d'avoir bien voulu mettre à la disposition du Bureau International de l'Heure les fonds nécessaires à l'élaboration des calculs et à la publication des deux fascicules précités (I60,000 francs français).

Elle recommande la poursuite de la publication sous la même forme pour les soixante et onze stations de longitude ayant pris part à l'opération.

Elle demande, après étude des devis d'impression qui lui ont été soumis, qu'une allocation annuelle de $375^{\circ}$ francs or soit mise pendant trois années consécutives, par chacune des Unions Astronomique et Géodésique-Géophysique, à la disposition du Président de la Commission mixte.

Cette publication consacrera ainsi l'Opération de I933, en fixant avec précision la position en longitude de soixante et onze stations à la surface de la Terre. 
D'autre part, aucune variation bien nette en longitude n'étant révélée par la comparaison des résultats de I933 avec ceux de I926, la Commission estime qu'il n'y a pas à refaire une opération des longitudes avant un délai d'au moins dix ou quinze ans, qu'il appartiendra aux deux Unions de fixer au cours d'une de leurs prochaines Assemblées Générales.

\section{Commission r9. (VARIATION DES LATITUDES)}

I. La réduction définitive du mouvement du pôle pour la période I93r.o jusqu'à 1936.06 n'a pas été publiée par M. Kimura. La Commission prie donc l'Union Astronomique d'accorder une subvention spéciale de 350 livres sterling pour cette publication.

2. La Commission émet le vœu que la subvention usuelle soit accordée pour les travaux du Bureau central, sous la direction du professeur Carnera.

3. La Commission demande qu'on exprime sa reconnaissance envers le professeur Carnera pour l'énergie, le zèle et les hautes connaissances scientifiques qu'il a montres dans l'élaboration des observations, et l'en remercie vivement.

\section{Commission 20. (Petrtes Plank̀tes, comètes et Satellites)}

L'Union recommande la continuation des recherches du professeur Leuschner sur les petites planètes, qui sont très utiles et sont caractérisées par leur très grande précision.

\section{Commission 22. (ÉTOILES FILANTES)}

I. La Commission insiste très respectueusement à nouveau pour que le Gouvernement Français veuille bien prendre des mesures pour explorer le site de la grande masse météorique de Chinguetti (Adrar).

2. La Commission suggère que quelque observatoire de l'hémisphère austral consacre une partie de son activité à l'étude des étoiles filantes, de préférence un observatoire de la Nouvelle-Zélande, où des travaux de ce genre ont déjà été effectués avec un succès marqué depuis plusieurs années.

3. La Commission félicite l'Association Astronomique Tchécoslovaque au sujet de l'achèvement de son atlas céleste gnomonique à l'usage des observateurs d'étoiles filantes, et recommande que cet atlas soit publié aussitôt que possible et au moindre prix de vente.

La Sous-Commission de la Lumière zodiacale et des phénomènes analogues a voté la résolution suivante:

4. Que des observations simultanées de la lumière zodiacale sur des plaques étalonnées soient entreprises dans quelque station équatoriale, en connexion avec les travaux du professeur Donitch à Assouan.

\section{Commission 23. (CARTE DU CIEI)}

I. La Commission considérant que les sept volumes du Catalogue photographique dont les observatoires d'Hyderabad, d'Edimbourg et d'Oxford avaient accepté la charge à la place des observatoires de Potsdam et de Rio de Janeiro, sont maintenant prêts pour l'impression, et considérant que les fonds mis en réserve par l'Union sont insuffisants pour couvrir les frais de leur impression, demande instamment à l'Union Astronomique de lui continuer jusqu'à l'achèvement de la publica- 